\title{
Design, test, and operation of new tapered stripline injection kickers for the $e^{+} e^{-}$collider DA $\Phi$ NE
}

\author{
David Alesini, Susanna Guiducci, Fabio Marcellini, and Pantaleo Raimondi \\ INFN Laboratori Nazionali di Frascati, P.O. Box 13, I-00044, Frascati (Roma), Italy
}

(Received 23 December 2009; published 29 November 2010)

\begin{abstract}
For the injection upgrade of the $\Phi$ factory DA $\Phi$ NE, new fast stripline kickers have been designed. They can operate with very short pulse generators to perturb only the injected bunch and the two stored adjacent ones. The design is based on tapering the striplines to simultaneously obtain low beam coupling and transfer impedances, excellent uniformity of the deflecting field, and better matching between the strip and the pulse generators. It has been done using 2D and 3D electromagnetic codes (SUPERFISH and HFSS). The kickers have been constructed, tested, and installed in the collider. Measurements of the reflection coefficient at input ports and of the longitudinal and transverse beam coupling impedance have been also performed to characterize the structure and have been compared to the simulation results. A circuital model of general tapered striplines for a first order estimation of the transfer and longitudinal beam coupling impedances is also presented. Finally operational performances are described, pointing out the problems which occurred and the flexibility of the stripline structures that worked with both the short and with the previously installed long pulse generators and have been used as an additional damping kicker to improve the efficiency of the horizontal multibunch feedback system. This system is also a demonstration of the operation of fast kickers with similar characteristics as those for the International Linear Collider (ILC) damping rings (DRs).
\end{abstract}

DOI: 10.1103/PhysRevSTAB.13.111002

PACS numbers: 29.27.Ac, 29.20.db, 41.85.Ar

\section{INTRODUCTION}

The Frascati $\Phi$ factory DA $\Phi$ NE [1] is a double ring, high luminosity collider working at the energy of the $\Phi$-meson resonance (1.02 GeV in the center of mass). Few relevant DAФNE parameters achieved in the last run dedicated to the experiment SIDDHARTA [2] are summarized in Table I.

The injection system of the collider is described in detail in [3]. The bunches coming from the damping ring are injected into each ring through the injection kickers 1 (Fig. 1). The second kicker just compensates the oscillations in the stored bunches caused by the injection kick. Old injection kickers were realized with two coils $[4,5]$ fed by high voltage (HV) pulsers based on thyratron switches. The length of such a pulse was approximately $150 \mathrm{~ns}$ and about 60 over the 110 stored bunches were perturbed during injection. Also the uniformity of the deflecting field as a function of the transverse coordinate was dominated by the magnetic field distribution of the two coils. The transverse deflecting force had a pseudosinusoidal behavior as a function of the horizontal coordinate with two zeros of the transverse force under the coils at about $\pm 2.5 \mathrm{~cm}$ from the pipe axis.

The new kickers have been realized to operate with new fast pulse generators recently available on the market $[6,7]$. Compared to the previous devices, the new system has the following main features: possibility to implement injection with much shorter pulse ( $\approx 12 \mathrm{~ns}$ instead of $\approx 150 \mathrm{~ns}$ ); better uniformity of the deflecting field as a function of the transverse coordinates; and lower beam impedance and possibility of higher repetition rate $(50 \mathrm{~Hz})$. The main parameters of the new kickers are given in Table II.

The kicker is basically a two stripline structure [9], where both the striplines and the surrounding chamber have been tapered. Each transverse section has constant $50 \Omega$ impedance to match the output impedance of the high voltage pulse generator. The field flatness has been obtained with a proper choice of the ratio between the length of tapered and straight sections, while the beam impedance reduction is the result of the tapering. From measurements presented in the following section, the structure turns out to be higher order mode (HOM) free.

Stripline injection kickers have been already proposed and adopted for fast injection and extraction in damping rings (DRs). The structures adopted in Accelerator Test Facility [10] or proposed for the TESLA DR [11], as

TABLE I. DAФNE parameters (SIDDHARTA runs 20082009).

\begin{tabular}{lcc}
\hline \hline Energy & $E[\mathrm{MeV}]$ & 510 \\
\hline Maximum beam current & $I_{M}[A]$ & $\approx 2.2\left(e^{-}\right)$ \\
& & $\approx 1.1\left(e^{+}\right)$ \\
Number of colliding bunches & $N_{b}$ & 110 \\
rf frequency & $f_{\mathrm{rf}}[\mathrm{MHz}]$ & $\approx 368.67$ \\
rf voltage & $V_{\mathrm{rf}}[\mathrm{kV}]$ & $150-170$ \\
Harmonic number & $h$ & 120 \\
Bunch spacing & $T_{B}[\mathrm{~ns}]$ & $\approx 2.7\left(=1 / f_{\mathrm{rf}}\right)$ \\
Maximum luminosity & $L\left[\mathrm{~cm}^{-2} \mathrm{~s}^{-1}\right]$ & $\approx 4.5 \times 10^{32}$ \\
\hline \hline
\end{tabular}




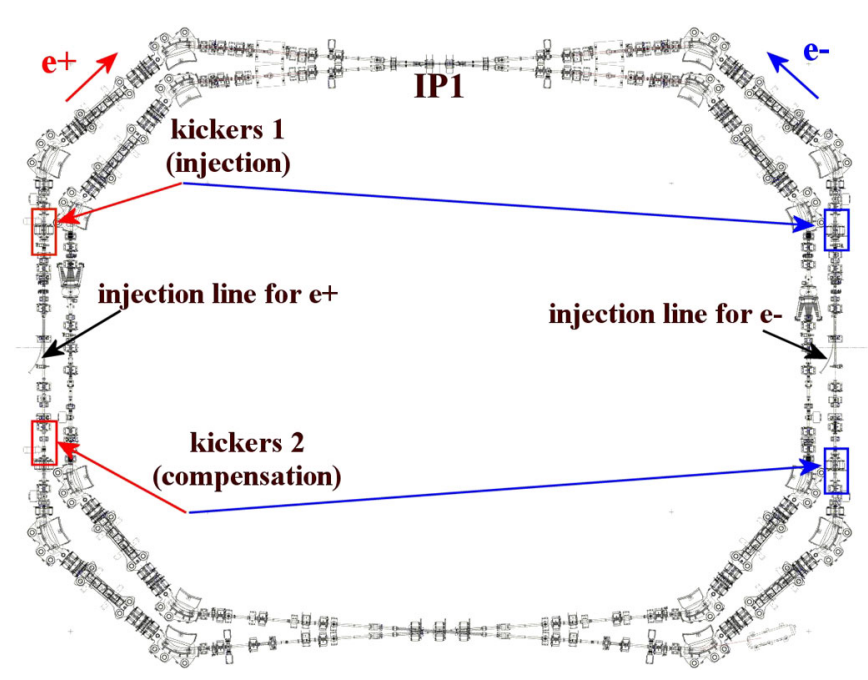

FIG. 1. Sketch of the DAФNE rings with injection system.

example, have constant impedance and constant profile stripline sections. In those cases the uniformity of the deflecting field has been increased by a proper shaping of the electrode transverse profile. The proton storage ring at LANL [12] had tapered striplines for proton extraction of about 4 meters but, in this case, the structure was tapered to match the beam size variation along the electrode and the electrode was offset in a direction to follow the displaced beam. The novelty of the new DAФNE kickers with respect to all these devices is the introduction of the tapered striplines to simultaneously obtain low beam coupling and transfer impedances, excellent uniformity of the deflecting field, and better matching between the strip and the pulse generators, as described in the paper. From this point of view, the new injection system represents also a test and a research and development activity of one of the most challenging issues of the ILC, i.e., the injection/extraction kickers for the DR [13]. Even if the required repetition rate and pulse stability of the ILC-DR kickers are different from the DAФNE ones, they have common requirements [14]: short pulse length (this minimizes the bunch distance and hence the DR circumference), good uniformity and high strength of the integrated deflecting field, and impedances of the structure as low as possible. The comparison between the DAФNE and ILC-DR parameters is reported in Table II.

In the first section of this paper the design of the new injection kickers is illustrated. In the second section we discuss the rf measurements, the high voltage tests, and the operational experience after their installation in the DAФNE collider. The advantages in introducing the tapered striplines are discussed in the third section. A simple transmission line model that allows estimating the longitudinal deflecting field distribution and the beam transfer and coupling impedances of tapered striplines are illustrated in the fourth section. The conclusions are reported in the last section.

\section{DESIGN OF THE NEW KICKERS}

\section{A. General considerations on pulse length requirements}

Considering a general stripline kicker [9], the total transverse deflecting voltage integrated by a particle, as a function of the particle time arrival in the kicker, can be calculated knowing the kicker geometry and the input pulse shape. As discussed in detail in Sec. IV of this paper, it is given by the convolution between the kicker impulse response (i.e. the deflecting voltage, as a function of time, when the input pulse is a $\delta$ function) and the input pulse shape. In the ideal case, if the kicker has a constant transverse section, and the matching between the pulse generator and the kicker structure is perfect, the kicker impulse response is rectangular as shown in Fig. 2(a) (assuming the particle velocity equal to $c$ ). The form factor

TABLE II. Parameters of the new DAФNE kickers and ILC kickers (nominal parameters [8]).

\begin{tabular}{lcc}
\hline \hline Parameter & DAФNE & ILC \\
\hline Total deflecting voltage $V_{T}[\mathrm{MV}]$ & 2.5 & 3.8 \\
Total deflection angle [mrad] & 5 & 0.76 \\
Rise time of the kicker pulse [ns] & $<6$ & $<6$ \\
Decay time of the kicker pulse [ns] & $<6$ & $<6$ \\
Flattop kicker pulse [ns] & $>0.5$ & $>0.12$ \\
Horizontal beam stay clear at kicker [mm] (diameter) $[\mathrm{mm}]$ & $>50$ & $>52$ \\
Bunch length of injected bunches $\sigma_{B}[\mathrm{~mm}]$ & $\approx 35$ & $\approx 11$ \\
Relative amplitude jitter & $<5 \%$ & $<0.065 \%$ \\
Maximum repetition rate [Hz] & 50 & $3 \times 10^{6}$ \\
Number of injected bunches & 120 & 2625 \\
Kicker electrical length [m] ${ }^{\mathrm{a}}$ ) & $\approx 0.85$ & $\approx 0.85$ \\
\hline \hline
\end{tabular}

${ }^{\text {a }}$ As discussed in Sec. II A, once we have fixed the rise, decay, and flattop time of the kicker pulse, the kicker electrical length depends on the rise and decay time of the input pulse. In this calculation we have considered them equal to 300 ps. 


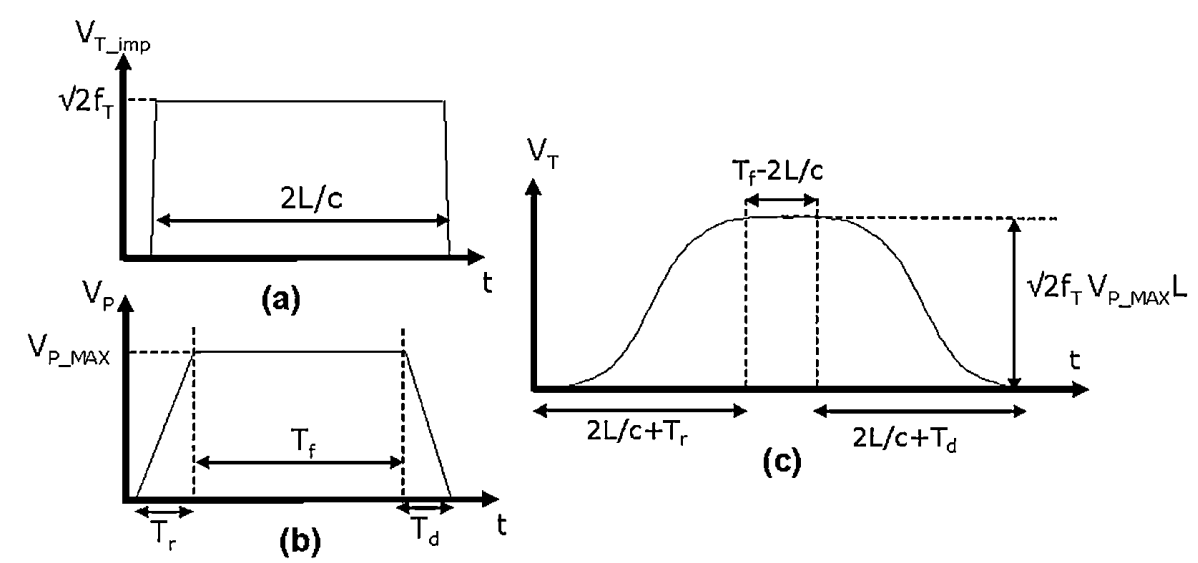

FIG. 2. (a) Ideal kicker impulse pulse response. (b) Simplified input pulse profile. (c) Total deflecting field as a function of time assuming $T_{f}>2 L / c>T_{r, d}$.

$f_{T}$ is related to the kicker geometry as discussed in Sec. IV and depends on the geometry of the striplines. If we consider an input pulse profile of the type shown in Fig. 2(b), the total transverse deflecting voltage integrated by a particle as a function of the particle time arrival is shown in Fig. 2(c) assuming of $T_{f}>2 L / c>T_{r, d}$. This simple model allows a first evaluation of the input pulse and kicker requirements in terms of rise/decay time $\left(T_{r, d}\right)$, flattop time $\left(T_{f}\right)$, kicker length $(L)$, and maximum input voltage $\left(V_{P_{-} \max }\right)$.

\section{B. General considerations on transverse field profile properties}

A cross section of a general circular stripline (one quarter of the structure) is sketched in Fig. 3 with the electric and magnetic field lines of the TEM deflecting mode. The

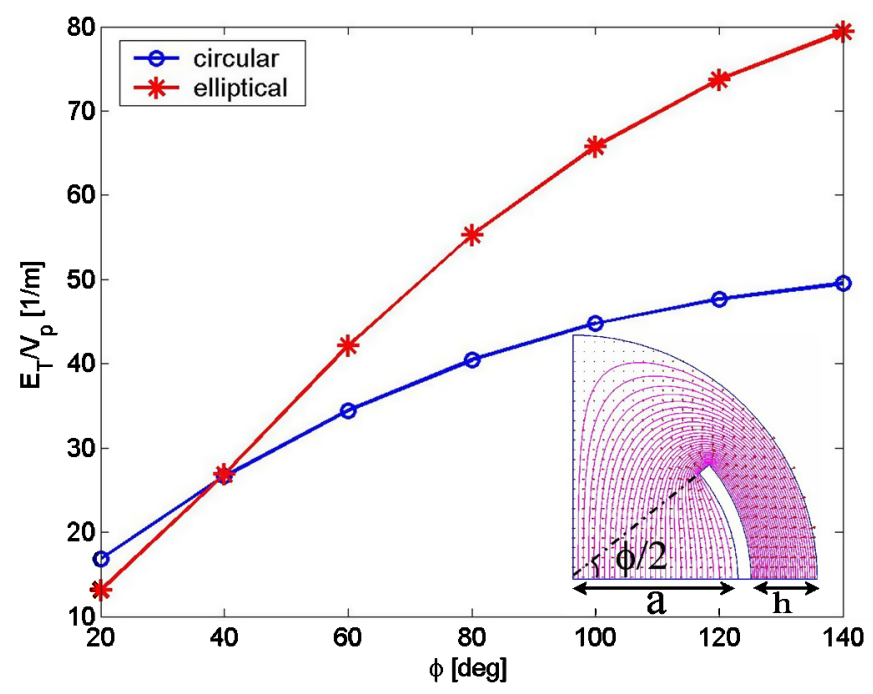

FIG. 3. Total equivalent deflecting field $E_{T}$ (normalized to the voltage per strip) at the center of the structure as a function of the coverage angle $\phi$ (stripline half-aperture $a=25 \mathrm{~mm}$ ). mode can be excited by feeding the two strips with opposite voltages while, to have a net deflection, the particle beam has to travel in the opposite direction with respect to the TEM wave [9]. For a given stripline aperture $(a)$ it is possible to calculate the total equivalent deflecting field $E_{T}$ (that takes into account the contribution of both electric and magnetic fields according to the Lorentz force) at the center of the structure, normalized to the input voltage per strip $V_{P}$, as a function of the coverage angle $\phi$. The result obtained with SUPERFISH [15] is plotted in Fig. 3 assuming $a=25 \mathrm{~mm}$ and the impedance of each strip equal to $50 \Omega$. As expected, the intensity of the deflecting field increases if $\phi$ increases. But, on the other hand, also the distance between the strip and the vacuum chamber itself has to be increased to maintain the strip impedance constant (for example, $\phi=140^{\circ}$ needs $h=$ $50 \mathrm{~mm}$ ). It is straightforward to note that $E_{T} / V_{P}$ is exactly equal to $\sqrt{2} f_{T}$.

The normalized deflecting field as a function of the coverage angle for an elliptical strip profile (with a ratio between the two axes equal to 1.5) is given in the same figure and compared to the case of a circular strip. For large coverage angles, the efficiency of an elliptical stripline is larger than the circular one because the strip is closer to the center of the pipe.

Another important point to be taken into account is the behavior of the deflecting field as a function of the transverse coordinates ( $x$ horizontal, $y$ vertical). The plot of the deflecting field normalized to its value at the center of the pipe, as a function of the horizontal coordinate, is given in Fig. 4(a) for three different values of the coverage angle. The three cases show a different behavior: for small values of $\phi$, the deflecting field increases with $x$ while, for large values of $\phi$, it decreases. This comes by the fact that, for small values of the coverage angle, the field generated by the strip is similar to that of a simple wire, while, for large values there is a shielding effect of the deflecting field given by the strip itself. The optimum case is represented 

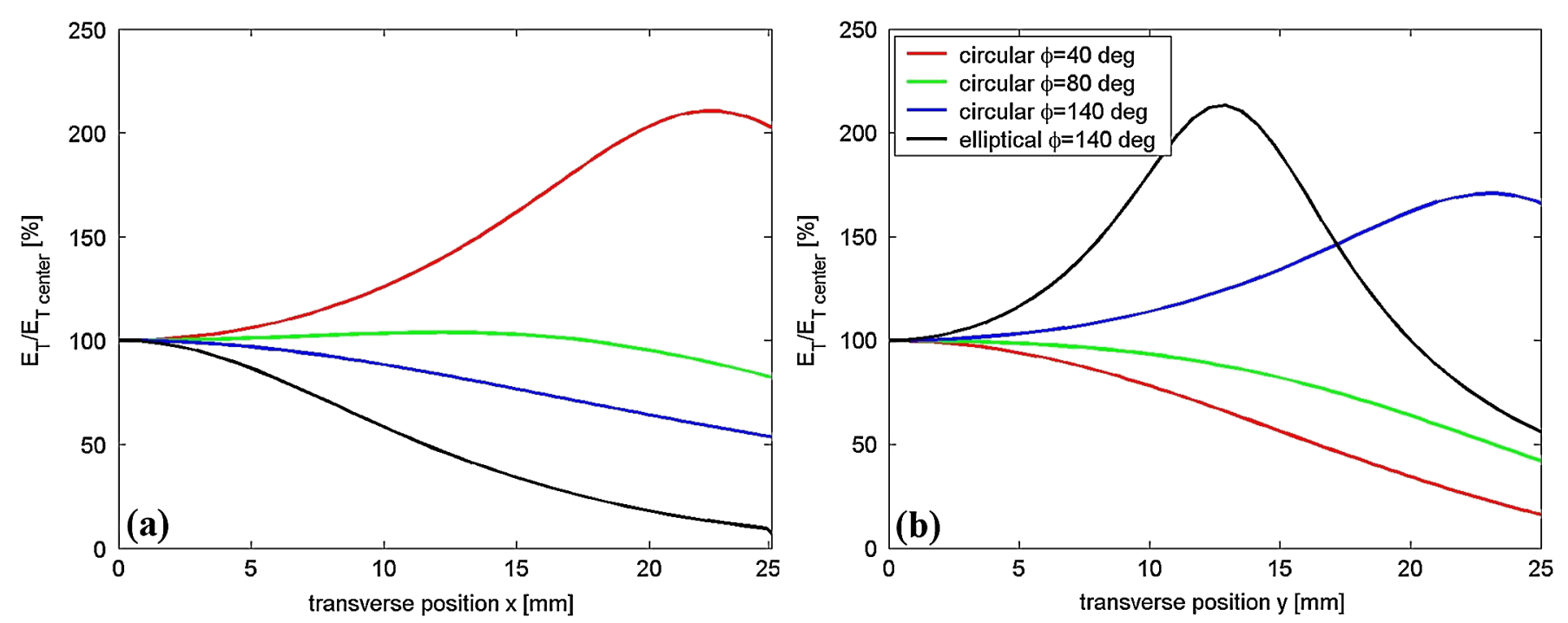

FIG. 4. Deflecting field normalized to its value at the center of the pipe, as a function of the transverse coordinates: (a) horizontal ( $x$ ); (b) vertical $(y)$.

by $\phi \cong 80 \mathrm{deg}$ even if, also in this case, the variation of the deflecting field as a function of the horizontal coordinate can be few tens of percent inside the good field region. In Fig. 4(b) we have plotted the same quantity as a function of the vertical coordinate $y$. In this case the behavior is opposite: for small values of $\phi$ the intensity decreases when the distance increases, while, for large values of $\phi$, the edge effects of the strip give a local increase of the field.

The elliptical strip case is reported in the same figures for comparison. In this case the deflecting field strongly decreases if we move from the center of the pipe toward the strip following the $x$ direction because of the shielding effect of the strip itself and it strongly increases if we move from the center toward the $y$ direction because of the edge effect on the stripline.

\section{Design of the stripline injection kickers}

The design of the kickers is based on the idea to properly taper the striplines and the surrounding vacuum chamber. The sketch of a tapered geometry is given in Fig. 5. Each transverse section should have constant impedance in order to avoid reflections of the input pulse.

The tapered scheme is applicable to both circular and elliptical geometries and allows one to: (a) reduce the broadband beam coupling impedance of the device since the discontinuity of the striplines is mitigated by the introduction of the tapers; (b) improve the deflecting field quality obtaining a uniform transverse deflection as a function of the transverse coordinate (horizontal, in particular); (c) obtain a better matching between the generator and the kicker structure at high frequency avoiding multiple reflections of the deflecting pulse; and (d) reduce the beam transfer impedance. Concerning the second point, it has been already shown in the previous paragraph that the uniformity of the deflecting field as a function of the transverse coordinate depends on the coverage angle. The length of the tapers with respect to the central region can be therefore optimized in order to obtain a uniform integrated deflecting field as a function of the horizontal coordinate $x$. The sections with small coverage angle, in fact, compensate the reduction of the deflecting field near the strip, given by the large coverage angle of the central part of the kicker.

Better matching between the pulse generator and the kicker structure is assured by reducing the stripline section and placing it very close to the kicker vacuum chamber in the coaxial-stripline transition region. This reduces also the beam coupling and transfer impedances of the device [8] as shown in Sec. IV.

To reduce the broadband impedance of the whole accelerator, an elliptical-like geometry has been chosen to have

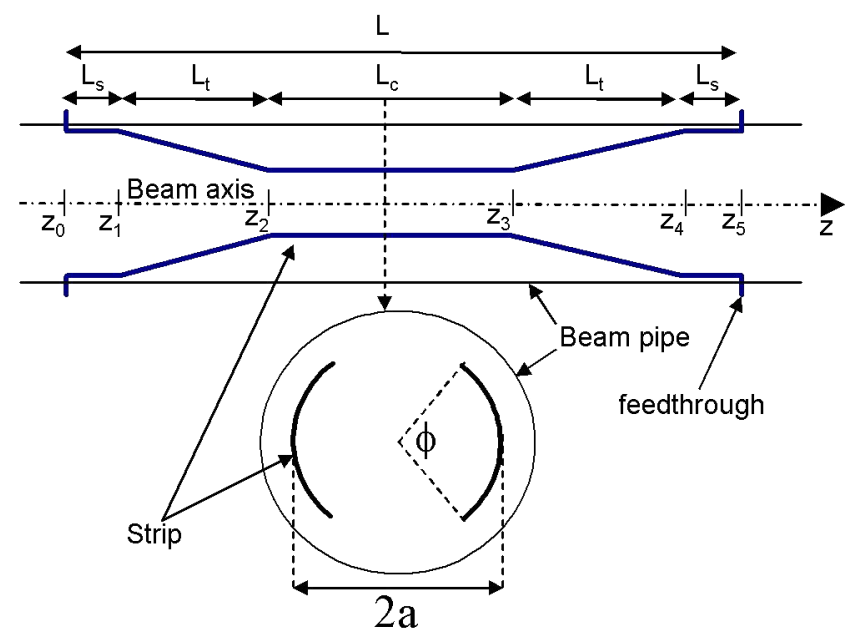

FIG. 5. Sketch of a tapered stripline kicker. 
a minimum variation of the vertical dimension of the beam pipe between the dipole and the injection regions of DAФNE. The general cross section of the kicker is sketched in Fig. 6(a) and the final optimized kicker dimensions are shown in Fig. 6(b): (a) each section of the kicker has the same $D h$ in order to not have a transverse modulation of the horizontal kicker vacuum chamber; (b) the value of $a, b$, and $t$ are the same for each section; (c) the $D$ and $D v$ dimensions have a linear modulation along the kicker; (d) the value of $\phi$ is progressively increased along the kicker up to $180^{\circ}$ (in the central part of the kicker) maintaining a constant $50 \Omega$ impedance in each section (equal to the output impedance of the pulsers); and (e) the values of $a$ and $b$ have been optimized together with the length of the central part of kicker and tapers in order to achieve at the same time an optimum deflecting field uniformity and a total electrical length of the kicker compatible with the bunch spacing and pulse length.

The integrated deflecting field as a function of the horizontal and vertical coordinates is shown in Figs. 7(a) and 7 (b), respectively. It has been obtained in two different ways: simulating 2D profiles at different longitudinal positions with Poisson-SUPERFISH and constructing the 3D map of the total deflecting field by interpolating the profiles at the different sections [8] and with the 3D code HFSS [16] by simulating the entire structure. In particular, the $2 \mathrm{D}$ procedure allowed one to strongly reduce the computational time needed to optimize the geometry of the structure and it is in good agreement with the final 3D (time consuming) simulations. The deflecting field in the center of the

(a)

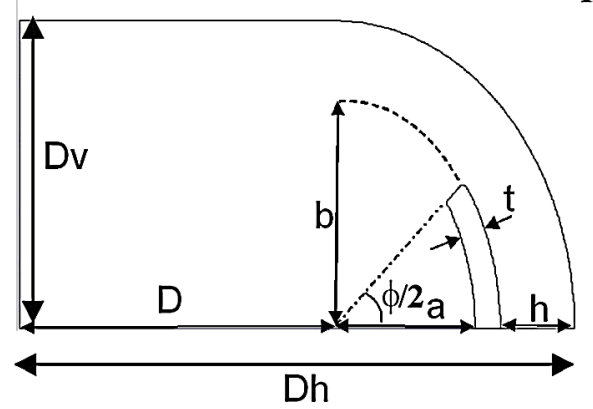

(b)

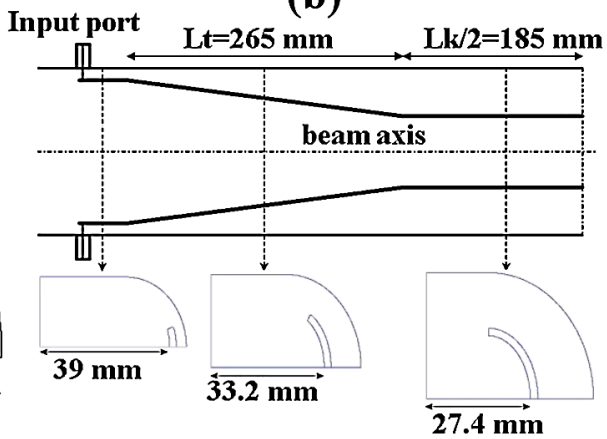

FIG. 6. (a) Generic cross section of the DAФNE kicker. (b) Final optimized dimensions of the kicker (half structure).
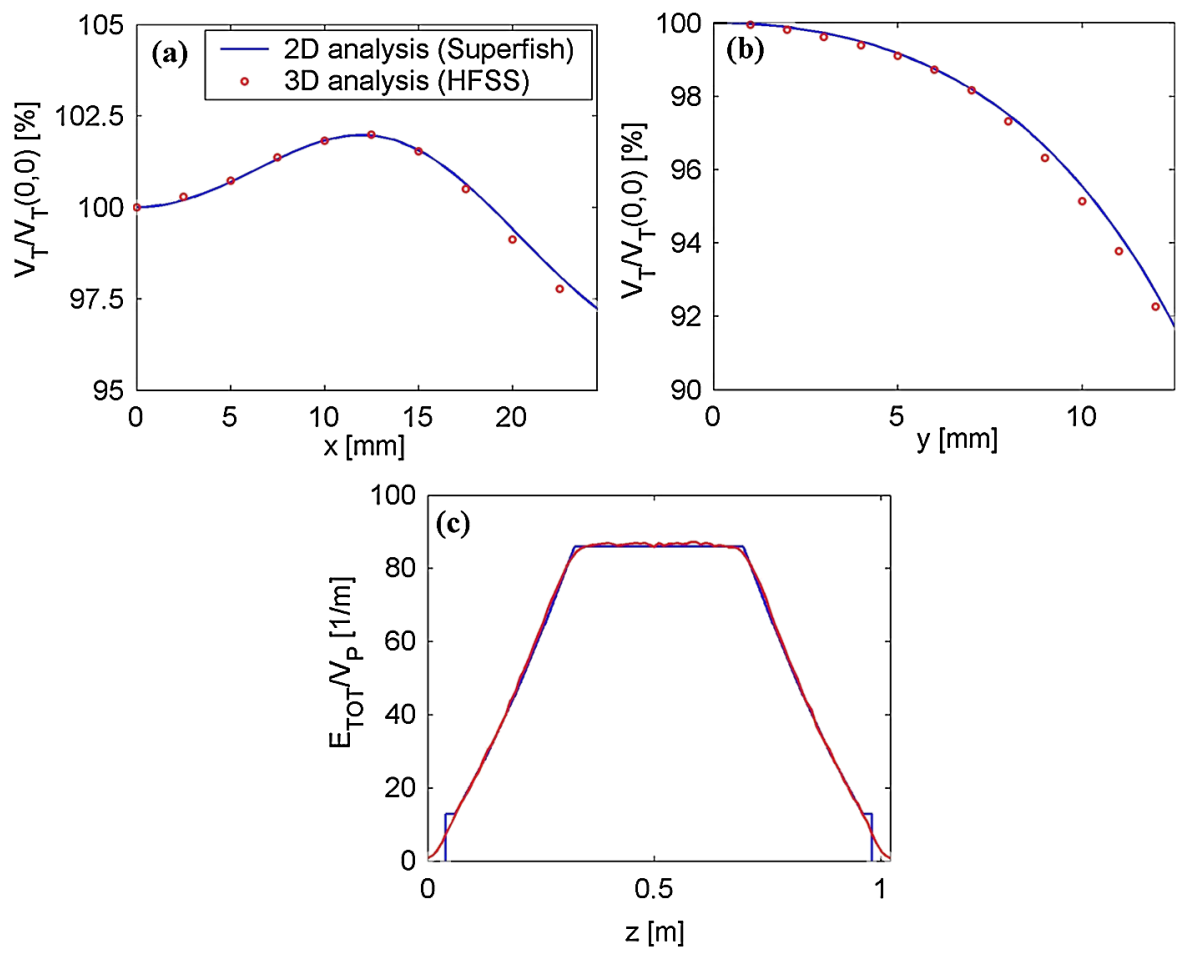

FIG. 7. Integrated deflecting field as a function of the horizontal (a) and vertical (b) coordinates. (c) Deflecting field in the center of the structure as a function of the longitudinal coordinate. 

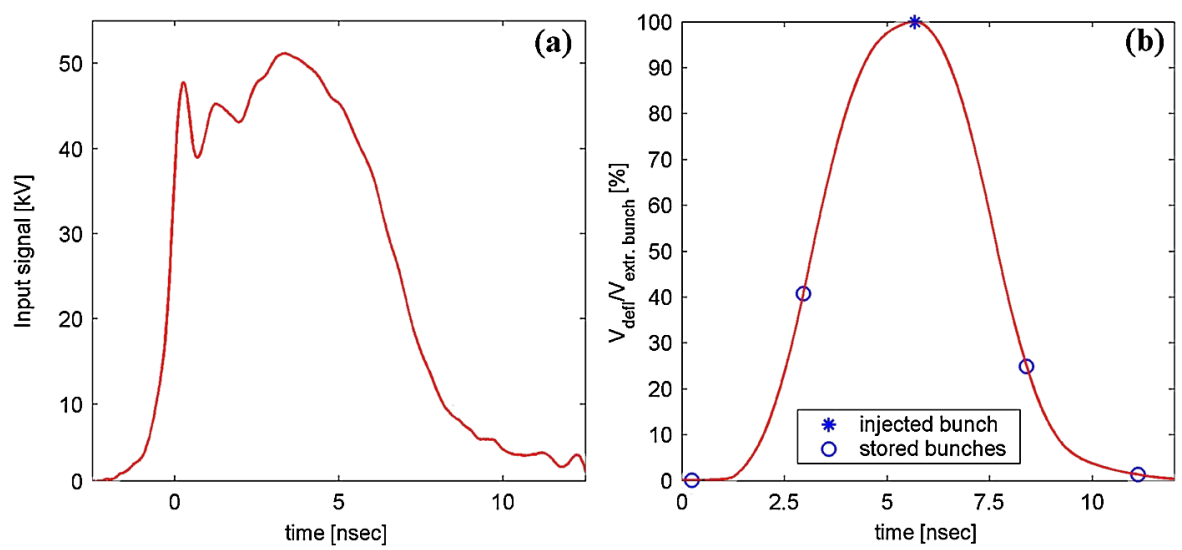

FIG. 8. (a) High voltage input pulse and related total deflecting field seen by bunches as a function of time arrival in the kicker structure (b).

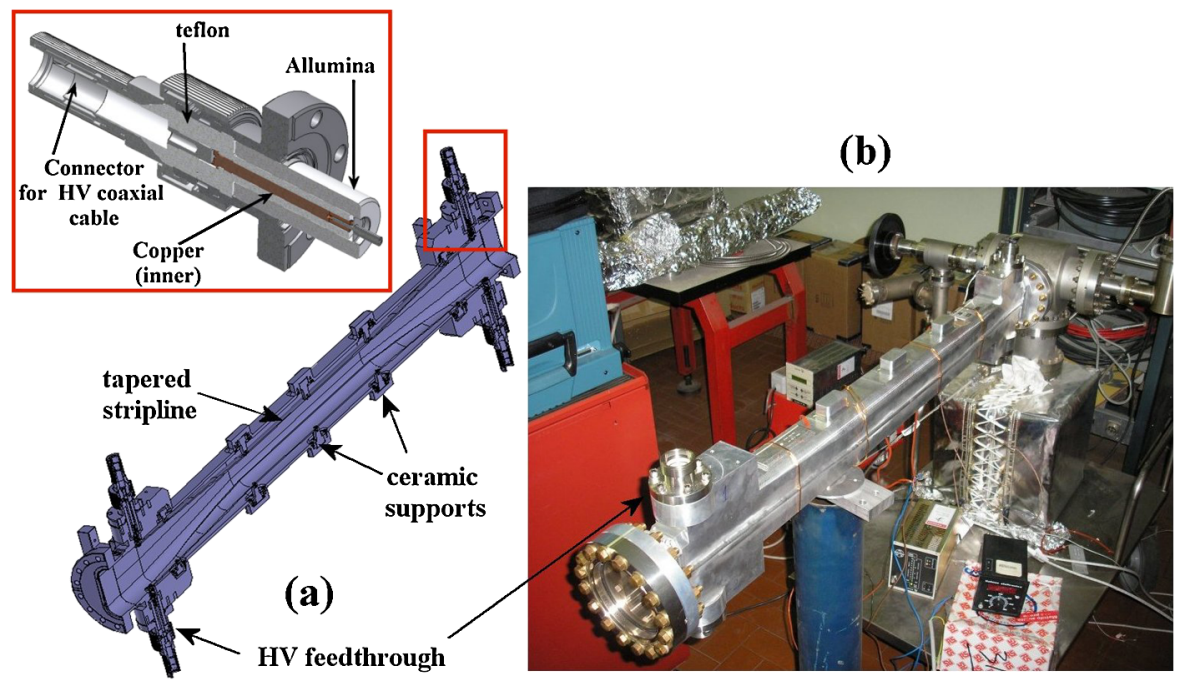

FIG. 9. (a) Final mechanical drawing of the kicker with a detail of the high voltage feedthrough. (b) Picture of the final realized kicker.

structure as a function of the longitudinal coordinate obtained by HFSS and compared with the $2 \mathrm{D}$ analysis is given in Fig. 7(c).

The whole kicker dimensions including the length of the tapers have been designed to have an integrated deflecting voltage along the kicker of $2.5 \mathrm{MV}$ with a maximum input voltage per strip of $45 \mathrm{kV}$.

The typical high voltage input pulse signal is given in Fig. 8(a). The pulse has a very short rise time (of about $300 \mathrm{ps}$ ), a longer decay time (of about $2.5 \mathrm{~ns}$ ) with a flattop time of about $5 \mathrm{~ns}$. From this profile it is possible to calculate the total deflecting field as a function of the bunch time arrival in the kicker structure. The result is shown in Fig. 8(b).

The final mechanical drawing (cross section) of the kickers and a picture of the realized kicker are shown in Figs. 9(a) and 9(b), respectively. The strip is sustained by ceramic supports. Since commercial $50 \Omega$ high voltage feedthroughs do not exist, they have been developed at LNF. The detail of their mechanical drawing is still reported in Fig. 9. Also the high voltage $50 \Omega$ loads have been developed at LNF.

\section{RF TEST AND OPERATIONAL EXPERIENCE}

\section{A. The rf measurements and HV tests}

Before installation in the DAФNE accelerator, the new kickers were tested and measured in the laboratory [17]. High voltage tests with the $45 \mathrm{kV}-5$ ns pulse generator have been tried successfully.

Figure 10 shows the measured reflection coefficient $\left(S_{11}\right)$ at the input port of the kickers. It is quite small up to $\approx 400 \mathrm{MHz}$ (the pulse frequency spectrum does not extend beyond), but increases at higher frequencies. This result is confirmed by the HFSS simulations that demonstrated that the deterioration of $S_{11}$ is mainly due to the 


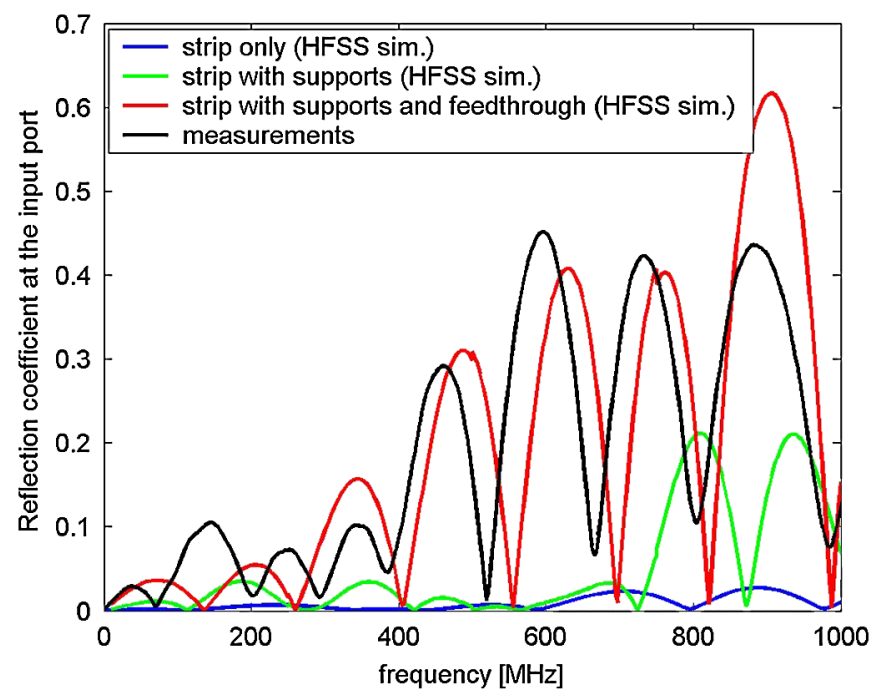

FIG. 10. Reflection coefficient at the input port: HFSS simulations and measurements.

feedthrough contributions. A research and development program on feedthrough with better frequency response is still in progress.

The wire method technique [18] has been used to fully characterize the kicker beam coupling and longitudinal impedances. The longitudinal coupling impedance has been measured with several different terminations at the four kicker ports: when $50 \Omega$ matches each port; when the two input ports are closed on short circuits and the output ports are on $50 \Omega$, and when the $50 \Omega$ are replaced by the real HV $50 \mathrm{Ohm}$ load built to be used for the operation. The first of these measurements gives the impedance of the stand-alone kicker, while the other two take account of mismatches introduced by connecting the pulsers (a short circuit is used to simulate the pulser in the worst possible condition). The same three sets of port terminations have been considered in the horizontal impedance measurements. HFSS simulations have been also performed and compared with the experimental results showing very good agreement [17].

The coupling impedance measurements and simulations have pointed out the absence of trapped HOMs in the longitudinal and horizontal planes when at least two ports are loaded on $50 \Omega$. Only with the input ports short circuited and the output ports terminated with HV loads some resonances have been measured. In the vertical plane four trapped HOMs $\left(\mathrm{TE}_{11 n}\right)$ were found even in ideal matching conditions. All these resonances have low beam coupling impedances [8,17] $\left(Z_{l}<30 \Omega\right.$ in the longitudinal plane, $Z_{t}<20 \mathrm{k} \Omega / \mathrm{m}$ in transverse planes) and, even in full coupling with beam spectrum lines, give instability growth rates well below the damping rates provided by the DAФNE feedback systems.

In conclusion, the new kickers give a very small negligible contribution to the DAФNE impedance budget [19].
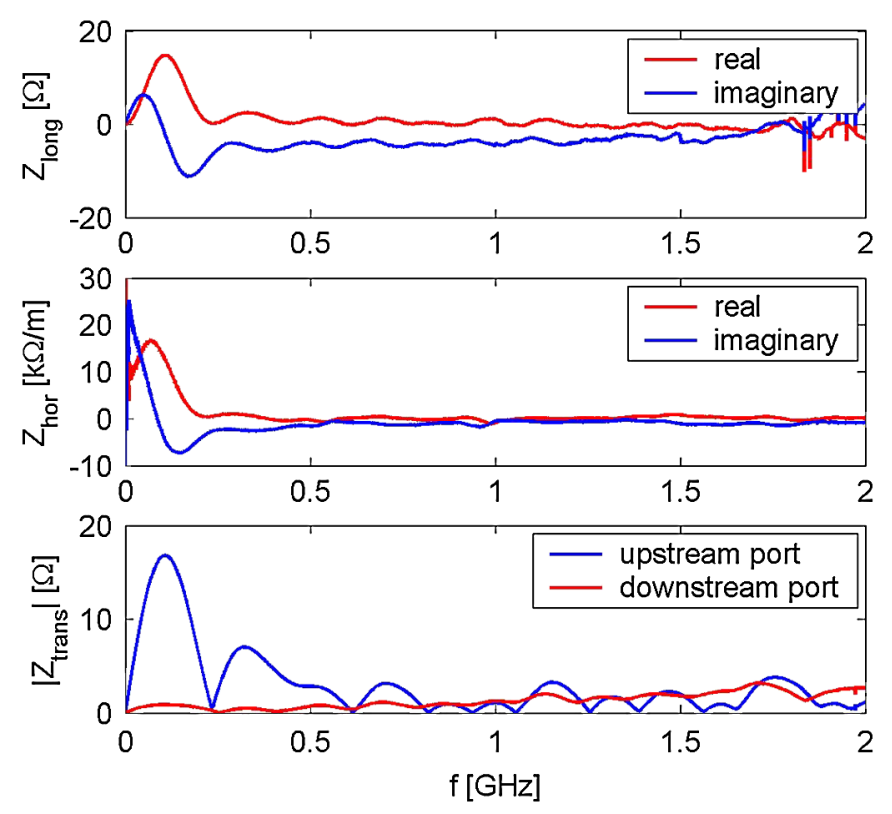

FIG. 11. Results of impedance measurements (wire technique).

Figure 11 shows, as an example, the measured longitudinal and horizontal impedances in the first matched case. The transverse impedance in the vertical plane was not measured because the kicker chamber is too narrow to place two wires and the related matching networks. In all the coupling impedance plots, the red lines refer to the real part and the blue lines to the imaginary part of the impedance. Since above the $\mathrm{TE}_{11}$ frequency cutoff of the coaxial line formed by the kicker chamber and the wire(s), coaxial $\mathrm{TE}_{11 n}$ resonances are usually measured, all the wire measurements have been limited at $2 \mathrm{GHz}$ [20].

In the same figure the measured transfer impedances of the input (downstream) and output (upstream) ports are also reported. Also in this case the results agree quite well with HFSS simulations.

The transfer impedance allows the evaluation of the peak voltage and the average power induced by the beam into the kicker ports for a given beam current. The maximum induced peak voltage on the upstream (output) ports is $<100 \mathrm{~V}$ with a $6 \mathrm{nC}$ bunch charge, while the average power induced on the ports is $<10 \mathrm{~W}$ with a $2 \mathrm{~A}$ beam [8].

The measurement results point out that there is a strong reduction of all impedances, with respect to the case of not tapered stripline kickers. This is also confirmed by the simple model introduced in the next section.

\section{B. Operational experience}

Four new kickers have been installed since November 2007 in the DAФNE storage rings. They always worked properly and never gave problems from the point of view of the devices themselves.

The injection with the high voltage fast pulse generators has been successfully tested. Figure 12 shows the rms 


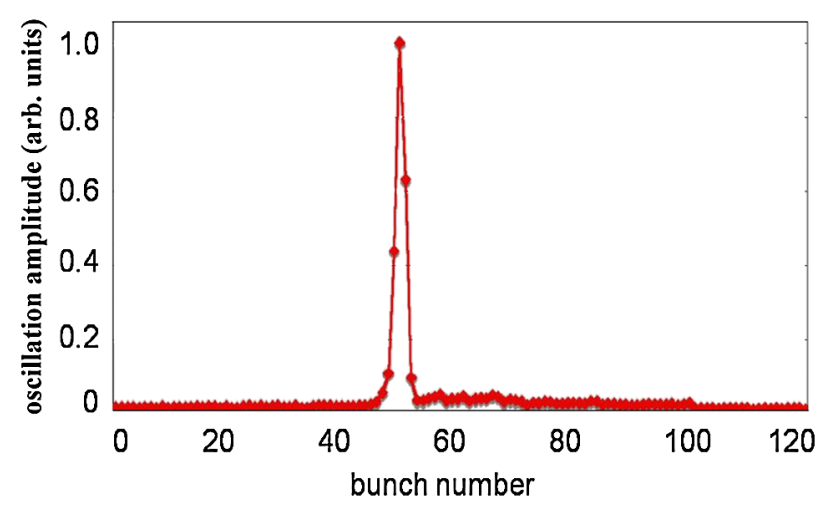

FIG. 12. Root-mean square relative oscillation amplitude measured by transverse feedback system of 100 stored bunches with kicker pulse centered on bunch 50 .

oscillation amplitude of 100 stored bunches with the kicker pulse centered on bunch 50 obtained with the horizontal digital feedback diagnostics [21]. As expected, bunches 49 and 51 take approximately half the kick maximum amplitude, while bunches 48 and 52 are only very little perturbed.

As a matter of fact, the operation with fast pulse generators has shown a poor reliability, even after repair and substitution of damaged parts with upgraded ones. The reasons of such poor reliability are still under study and radiation damages and beam-pulse interference (especially at high beam current) have been also considered. In any case we never had the possibility to use routinely and at the same time the eight fast pulsers.

Several different injection configurations have been therefore used.

For a great part of the operation time, the kickers worked with the old long pulse generators in both of the rings. In this configuration the two strips are connected in series and the kick is only given by the magnetic field one.

Also a different "hybrid" configuration has been successfully tested. In this case the two kinds of pulsers (long and fast) were used together on the same kicker, connecting each one to a different stripline. Figure 13 shows, as an example, the sum of the signal detected at the two stripline output ports of each kicker in this configuration. The difference between the fast and long pulses is very clear.

The new stripline kicker has been also used as an additional kicker for the horizontal feedback in the positron ring. One stripline of both kickers of the DAФNE positron ring has been connected with the old pulser for beam injection and the remaining stripline connected to the amplifiers of the feedback system. Thanks to this configuration, it has been possible to increase the stored current in the $e^{+}$ring to more than $1 \mathrm{~A}[1,21]$.

With the aim to overcome the problems related to the fast pulser reliability, it has been decided to try a different and more compact model that produces an output pulse having the same shape but a reduced amplitude from $45 \mathrm{kV}$

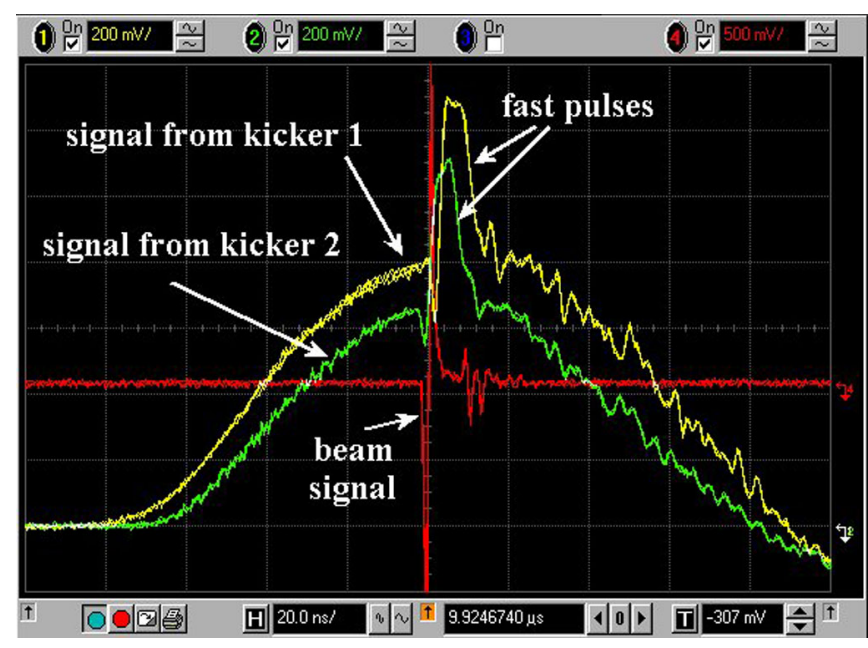

FIG. 13. The hybrid injection: long and fast pulses, from two stripline kickers, are observed in sum at the scope.

to $25-28 \mathrm{kV}$. In spite of the lower kick voltage, increasing the $\beta$ function in the kicker region and changing the beam orbit in the septa, the injection was possible as well. According to the hybrid scheme, two $25 \mathrm{kV}$ units were installed in the electron ring at the end of the SIDDARTHA run (September 2009-November 2009) and have been successfully tested up to the end of the run for about two months of operation without problems. Therefore in the next run starting on September 2010 we will install the $25 \mathrm{kV}$ units in both rings.

\section{ADVANTAGES OF TAPERED KICKERS}

The advantages in tapering the striplines of the kickers can be pointed out with simple electromagnetic simulations. Here we consider, as an example, the case of a kicker with the dimensions reported in Fig. 14(a) and we compare its performances with those of the not tapered striplines reported in Fig. 14(b). The two kickers give the same deflecting field for a given strip voltage and have the same electrical length. The longitudinal beam coupling impedance and the transfer impedance calculated by GDFIDL [22] in the two cases up to $20 \mathrm{GHz}$ are reported in Fig. 15. In both cases we have simulated one quarter of the structure with magnetic boundary conditions and matched ports using a mesh size of $0.25 \mathrm{~mm}$, a bunch length of $3 \mathrm{~mm}$, and a wake length of $100 \mathrm{~m}$.

The results show the strong reduction of the impedance and transfer impedance content when we have a tapered geometry. From HFSS simulation it is also easy to verify that the reflection coefficient at the input port is also reduced above $1 \mathrm{GHz}$. It is also important to remark that the calculation of the coupling and transfer impedances by HFSS simulating the beam with a wire, gives the same results as GDFIDL simulations within a $15 \%$ of error due to the wire approximation. Of course, the wire simulations 


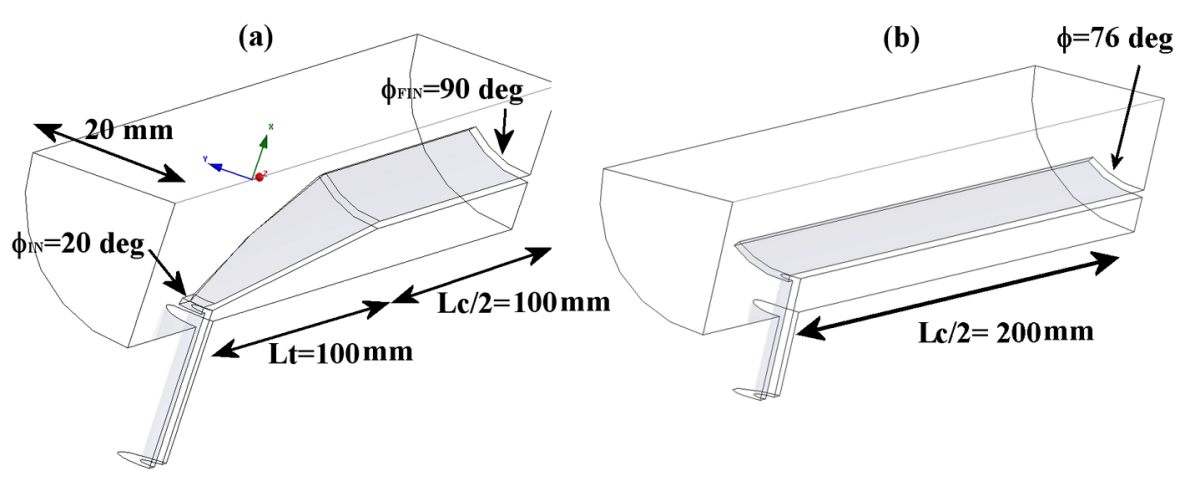

FIG. 14. GDFIDL simulated structures: (a) tapered; (b) not tapered.
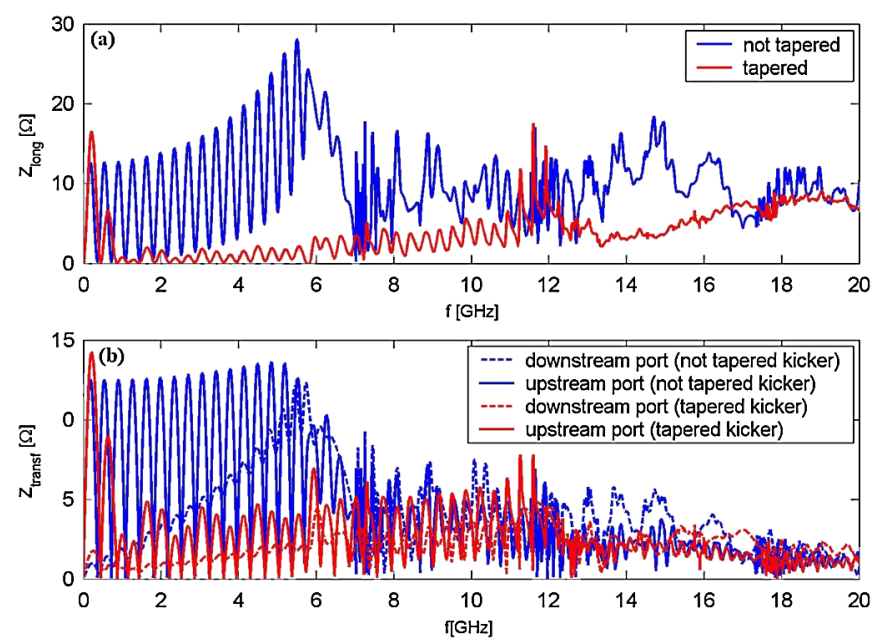

FIG. 15. The longitudinal beam coupling impedance and the transfer impedance calculated by GDFIDL.

are valid below the cutoff frequency of the beam pipe modes, that, in our example, are at $\sim 4.4 \mathrm{GHz}$ and $\sim 5.7 \mathrm{GH} z$ for the $\mathrm{TE}_{11}$ and $\mathrm{TM}_{01}$ modes, respectively.

\section{TRANSMISSION LINE MODEL FOR TAPERED STRIPLINE KICKERS}

In this section we will introduce a simplified transmission line model that allows estimating the tapered kicker efficiency and the beam transfer and coupling impedances.

\section{A. Transverse beam voltage}

The sketch of a tapered stripline with a circular cross section is given in Fig. 5. In the simplest case the tapered striplines have, at each longitudinal section, circular profiles with constant impedance and different coverage angles $\phi$.

To calculate the transverse beam force seen by the beam in correspondence of a certain $z$ coordinate, we can use the formula given in [23] that allows calculating the transverse electric field in each section as a function of the geometrical strip parameters. In the frequency domain, the total equivalent deflecting field at the longitudinal coordinate $z$, that takes into account the contribution of both electric and magnetic fields according to the Lorentz force and a propagating field with opposite direction with respect to the beam [9], is given by

$$
\tilde{E}_{T}=\frac{8}{\pi} \sin \left(\frac{\phi(z)}{2}\right) \frac{1}{2 a(z)} \hat{V}_{p} e^{j\left(\omega / v_{s}\right) z} e^{j \omega t},
$$

where $\hat{V}_{p}$ is the peak voltage per strip and $v_{s}$ is the velocity of the propagating field.

Equation (1) is referred to the case of two striplines with a distance $2 a$ exactly equal to the beam pipe diameter and assuming a relativistic factor of the beam $\beta_{B}=v_{B} / c \approx 1$ ( $v_{B}$ is the beam velocity).

Equation (1) can be generalized according to the theory illustrated in [9] in the following form:

$$
\tilde{E}_{T}=\sqrt{2} \underbrace{\frac{g_{T}(z)}{2 a(z)}}_{f_{T}(z)} \hat{V}_{p} e^{j\left(\omega / v_{s}\right) z} e^{j \omega t},
$$

where $g_{T}$ is a generic transverse coverage factor and $f_{T}$ is the so-called form factor of the strip.

The maximum total deflecting voltage seen by the beam is given by

$$
\begin{aligned}
V_{T} & =|\underbrace{\int_{0}^{L} E_{T}^{*} e^{j \omega\left[\left(1 / v_{s}\right)+\left(1 / v_{B}\right)\right] z} e^{j \omega t^{*}} d z}_{\tilde{V}_{T}}| \\
& =\sqrt{2} \hat{V}_{p}\left|\int_{0}^{L} f_{T}(z) e^{j \omega\left[\left(1 / v_{s}\right)+\left(1 / v_{B}\right)\right] z} e^{j \omega t^{*}} d z\right|,
\end{aligned}
$$

where $t^{*}$ is the relative time arrival of the beam in the kicker with respect to the propagating field and $\tilde{V}_{T}$ is the deflecting voltage in the frequency domain.

In the case of a strip without tapering Eq. (3) gives the well-known formula: 


$$
V_{T}=\sqrt{2} \hat{V}_{p} \bar{f}_{T} \frac{\sin \left[\frac{\omega L}{2}\left(\frac{1}{v_{s}}+\frac{1}{v_{B}}\right)\right]}{\frac{\omega}{2}\left(\frac{1}{v_{s}}+\frac{1}{v_{B}}\right)},
$$

where $\bar{f}_{T}$ is the constant form factor.

The transverse shunt impedance as defined in [9] is given by

$$
R_{T}=\frac{\left|\tilde{V}_{T}\right|^{2}}{2 P_{\mathrm{IN}}}=2 Z_{0}\left|\int_{0}^{L} f_{T} e^{j \omega\left[\left(1 / v_{s}\right)+\left(1 / v_{B}\right)\right] z} d z\right|^{2}
$$

where $Z_{0}$ is the characteristic impedance of each strip and $P_{\mathrm{IN}}$ is the input power in each stripline.

In the case of a strip without tapering, Eq. (5) gives the well-known formula [9]

$$
R_{T}=2 Z_{0}\left\{\bar{f}_{T} \frac{\sin \left[\frac{\omega L}{2}\left(\frac{1}{v_{s}}+\frac{1}{v_{B}}\right)\right]}{\frac{\omega}{2}\left(\frac{1}{v_{s}}+\frac{1}{v_{B}}\right)}\right\}^{2} .
$$

The impulsive kicker time response is the total voltage seen by the beam assuming an input voltage of the type

$$
V_{p_{-} \text {imp }}(t)=\delta(t)
$$

which corresponds to a propagating field of the type

$$
E_{T \text { imp }}(z, t)=\sqrt{2} f_{T}(z) \delta\left(z-L+t v_{s}\right) .
$$

In this case the total voltage seen by the beam as a function of its time arrival in the kicker $t^{*}$ can be simply obtained by

$$
\begin{aligned}
V_{T_{\text {imp }}}\left(t^{*}\right) & =\int_{0}^{L} E_{T \text { imp }}\left(z, t=\frac{L+t^{*} v_{B}}{v_{s}+v_{B}}\right) d z \\
& =\sqrt{2} f_{T}\left[L-L \frac{v_{s}}{v_{s}+v_{B}}+t^{*}\left(\frac{1}{v_{B}}+\frac{1}{v_{s}}\right)^{-1}\right] .
\end{aligned}
$$

From Eq. (9) it is straightforward to note that the impulsive time response in the case of a tapered kicker has a trapezoidal form, while, for a not tapered stripline, it has a rectangular form.

\section{B. Beam transfer impedance calculation}

Following the treatment illustrated in [24], the equivalent circuit of a uniform stripline excited by the beam is shown in Fig. 16(a). The stripline can be considered as a transmission line of length $L$ which is exposed to the beam electromagnetic field. The transverse dimensions of the stripline determine the characteristic impedance at any $z$. The strip is terminated on $Z_{1}$ and $Z_{2}$. The relativistic charged particle beam couples to the stripline and induces a current on each strip termination that is a fraction $g$ of the beam current [24].

In the circuit we have considered a single beam harmonic component of the type $I_{b}=I_{0} e^{-j\left(\omega / \beta_{B} c\right) z}=$ $I_{0} e^{-\gamma_{B} z}$, where $\beta_{B} c$ is the beam velocity.
The beam transfer impedances upstream and downstream, in the case of a uniform stripline, are given by [24]

$$
\begin{aligned}
Z_{\text {up }}(\omega)= & \frac{V_{\text {up }}(\omega)}{I_{0}}=g \frac{Z_{0}}{2}\left(1+\Gamma_{1}\right) \\
& \times \frac{1-\left(1+\Gamma_{2}\right) e^{-\gamma_{s} L} e^{-\gamma_{B} L}+\Gamma_{2} e^{-2 \gamma_{s} L}}{1-\Gamma_{1} \Gamma_{2} e^{-2 \gamma_{s} L}} \\
Z_{\mathrm{dn}}(\omega)= & \frac{V_{\mathrm{dn}}(\omega)}{I_{0}}=g \frac{Z_{0}}{2}\left(1+\Gamma_{2}\right) \\
& \times \frac{\left(1+\Gamma_{1} e^{-2 \gamma_{s} L}\right) e^{-\gamma_{B} L}-\left(1+\Gamma_{1}\right) e^{-\gamma_{s} L}}{1-\Gamma_{1} \Gamma_{2} e^{-2 \gamma_{s} L}},
\end{aligned}
$$

where $\gamma_{s}=\alpha+j \omega /\left(\beta_{s} c\right)$ is the propagation constant for a signal on the stripline and $\Gamma_{1,2}$ are the reflection coefficients at the input/output strip terminations given by

$$
\Gamma_{1,2}=\frac{Z_{1,2}-Z_{0}}{Z_{1,2}+Z_{0}}
$$

Looking at the transfer impedance expressions, it is important to remark that the coverage factor $g$ is the fraction of the intercepted beam image current at the stripline edges.

In the case of a tapered stripline, we suppose that the characteristic impedance is constant along the strip and that the tapering consists in a change of the coverage factor along the $z$ coordinate.

The equivalent circuit is reported in Fig. 16(b). In this case we have the two generators at the beginning and at the end of the structure corresponding to the intercepted beam current at the stripline edges, and distributed generators along the tapers. Assuming that the two tapers are equal, extending the formulas reported in [24], we can write

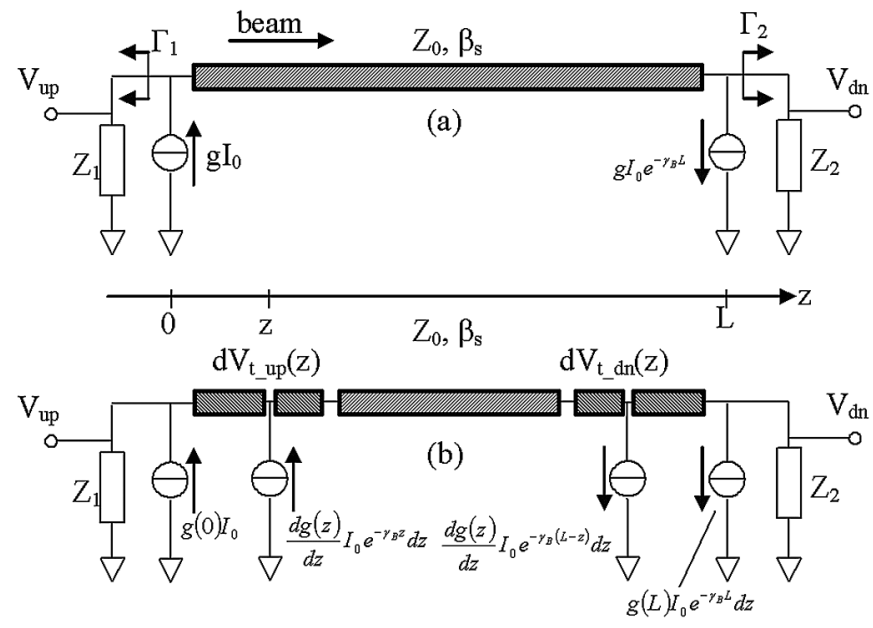

FIG. 16. Equivalent circuits of a stripline excited by the beam: (a) uniform stripline; (b) tapered stripline. 


$$
\begin{aligned}
Z_{\text {up }}(\omega)= & g(0) \frac{Z_{0}}{2}\left(1+\Gamma_{1}\right) \frac{1-\left(1+\Gamma_{2}\right) e^{-\gamma_{s} L} e^{-\gamma_{B} L}+\Gamma_{2} e^{-2 \gamma_{s} L}}{1-\Gamma_{1} \Gamma_{2} e^{-2 \gamma_{s} L}}+\frac{Z_{0}}{2} \int_{0}^{L_{t}} \frac{d g}{d z}\left(1+\Gamma_{1}\right) \\
& \times \frac{1-\left(1+\Gamma_{2} e^{-2 \gamma_{s} z}\right) e^{-\gamma_{s}(L-2 z)} e^{-\gamma_{B}(L-2 z)}+\Gamma_{2} e^{-2 \gamma_{s} z} e^{-2 \gamma_{s}(L-2 z)}}{1-\Gamma_{1} \Gamma_{2} e^{-4 \gamma_{s} z} e^{-2 \gamma_{s}(L-2 z)}} e^{-\gamma_{B} z} e^{-\gamma_{s} z} d z \\
Z_{\mathrm{dn}}(\omega)= & g(0) \frac{Z_{0}}{2}\left(1+\Gamma_{2}\right) \frac{\left(1+\Gamma_{1} e^{-2 \gamma_{s} L}\right) e^{-\gamma_{B} L}-\left(1+\Gamma_{1}\right) e^{-\gamma_{s} L}}{1-\Gamma_{1} \Gamma_{2} e^{-2 \gamma_{s} L}}+\frac{Z_{0}}{2} \int_{0}^{L_{t}} \frac{d g}{d z}\left(1+\Gamma_{2}\right) \\
& \times \frac{\left(1+\Gamma_{1} e^{-2 \gamma_{s}(L-2 z)} e^{-2 \gamma_{s} z}\right) e^{-\gamma_{B}(L-2 z)}-\left(1+\Gamma_{1} e^{-2 \gamma_{s} z}\right) e^{-\gamma_{s}(L-2 z)}}{1-\Gamma_{1} \Gamma_{2} e^{-4 \gamma_{s} z} e^{-2 \gamma_{s}(L-2 z)}} e^{-\gamma_{B} z} e^{-\gamma_{s} z} d z .
\end{aligned}
$$

In the case of a matched stripline with $\gamma_{B}=\gamma_{S}=\gamma$, we obtain

$$
\begin{aligned}
Z_{\text {up }}(\omega)= & g(0) \frac{Z_{0}}{2}\left(1-e^{-2 \gamma L}\right)+\frac{Z_{0}}{2} \\
& \times \int_{0}^{L_{t}} \frac{d g}{d z}\left(e^{-2 \gamma z}-e^{-2 \gamma(L-z)}\right) d z \\
& Z_{\mathrm{dn}}(\omega)=0 .
\end{aligned}
$$

Assuming a linear coverage factor along the tapers of the type

$$
g(z)=g(0)+\frac{g\left(L_{t}\right)-g(0)}{L_{t}} z
$$

we obtain

$$
\begin{aligned}
Z_{\text {up }}(\omega)= & g(0) \frac{Z_{0}}{2}\left(1-e^{-2 \gamma L}\right)+\frac{Z_{0}}{2} \frac{g\left(L_{t}\right)-g(0)}{L_{t}} \\
& \times \frac{1}{2 \gamma}\left[\left(1-e^{-2 \gamma L_{t}}\right)+e^{-2 \gamma L}\left(1-e^{2 \gamma L_{t}}\right)\right] .
\end{aligned}
$$

From previous expressions it is easy to note that, assuming a negligible coverage factor $g(0)$ at the beginning and at the end of the strip, the beam transfer impedances $Z_{\text {up }}$ can be reduced as much as one wants by increasing the taper length $L_{t}$. In the formulas it is also easy to find that the effect of the tapers is related to the second part of the expression and this term goes to zero reducing the wavelength. It is easy to show that comparison between the model and the simulations (GDFIDL or HFSS) has shown a quite good agreement.

\section{Beam coupling impedance}

Extending the calculation developed in $[9,24]$ to the tapered case, it is possible to evaluate the longitudinal beam coupling impedance from the formula:

$$
\begin{aligned}
Z_{L}(\omega)= & g^{*}(0) Z_{\text {up }}(\omega)+\int_{0}^{L_{t}} \frac{g^{*}(z) d V_{t_{-} \text {up }}(z, \omega)}{I_{0} e^{-\gamma_{B} z}} \\
& -\int_{L-L_{t}}^{L} \frac{g^{*}(z) d V_{t_{-} \mathrm{dn}}(z, \omega)}{I_{0} e^{-\gamma_{B} z}-g^{*}(L) Z_{\mathrm{dn}}(\omega) e^{\gamma_{B} L},}
\end{aligned}
$$

where $Z_{\text {up }}$ and $Z_{\text {dn }}$ are given in Eqs. (13) and (14) and $V_{t}$ up and $V_{t_{-} \mathrm{dn}}$ are the beam induced voltages in each position $z$ along the tapers [as shown in Fig. 16(b)]. In this case the coverage factor $g^{*}$ is double with respect to the coverage factors of the previous paragraph because of the presence of the two striplines.

Extending the calculation reported in [24] it is easy to find that

$$
\begin{gathered}
d V_{t_{-} \text {up }}(z, \omega)=\frac{d g}{d z} \frac{Z_{0}}{2}\left(1+\Gamma_{1} e^{-2 \gamma_{s} z}\right) \frac{1-\left(1+\Gamma_{2} e^{-2 \gamma_{s} z}\right) e^{-\gamma_{s}(L-2 z)} e^{-\gamma_{B}(L-2 z)}+\Gamma_{2} e^{-2 \gamma_{s} z} e^{-2 \gamma_{s}(L-2 z)}}{1-\Gamma_{1} \Gamma_{2} e^{-2 \gamma_{s}(L-2 z)} e^{-4 \gamma_{s} z}} I_{0} e^{-\gamma_{B} z} d z \\
d V_{t_{-} \text {dn }}(z, \omega)=\frac{d g}{d z} \frac{Z_{0}}{2}\left(1+\Gamma_{2} e^{-2 \gamma_{s} z}\right) \frac{\left(1+\Gamma_{1} e^{-2 \gamma_{s} z} e^{-2 \gamma_{s}(L-2 z)}\right) e^{-\gamma_{B}(L-2 z)}-\left(1+\Gamma_{1} e^{-2 \gamma_{s} z}\right) e^{-\gamma_{s}(L-2 z)}}{1-\Gamma_{1} \Gamma_{2} e^{-4 \gamma_{s} z} e^{-2 \gamma_{s}(L-2 z)}} I_{0} e^{-\gamma_{B} z} d z .
\end{gathered}
$$

If we consider the case of a perfect matched stripline with a linear coverage factor along the tapers and $\gamma_{B}=\gamma_{S}=\gamma$, we obtain 


$$
\begin{aligned}
Z_{L}(\omega)= & \frac{Z_{0}}{2}\left\{-g(0)^{2} e^{-2 \gamma L}+\frac{1}{2}\left[g\left(L_{t}\right)^{2}+g(0)^{2}\right]+g(0) \frac{g\left(L_{t}\right)-g(0)}{L_{t}} \frac{1}{2 \gamma}\left[\left(1-e^{-2 \gamma L_{t}}\right)+e^{-2 \gamma L}\left(1-e^{2 \gamma L_{t}}\right)\right]-g(0)\right. \\
& \left.\times \frac{g\left(L_{t}\right)-g(0)}{L_{t}} \frac{1}{4 \gamma} e^{-2 \gamma L}\left(e^{4 \gamma L_{t}}-1\right)-\left[\frac{g\left(L_{t}\right)-g(0)}{L_{t}}\right]^{2} e^{-2 \gamma L}\left[e^{4 \gamma L_{t}}\left(\frac{L_{t}}{4 \gamma}-\frac{1}{16 \gamma^{2}}\right)+\frac{1}{16 \gamma^{2}}\right]\right\}
\end{aligned}
$$

Comparisons between simulations and theoretical values shown that this approach can give a first evaluation of the beam coupling impedance. In the theoretical model is also easy to distinguish the effect of the tapers in the beam coupling impedance expression.

\section{CONCLUSIONS}

A new fast kicker has been designed for the injection upgrade of the $\Phi$ factory DA $\Phi N E$. Using a tapered stripline it has been possible to simultaneously reduce the impedance of the device and to improve the deflecting field quality. The design has been done using $2 \mathrm{D}$ and $3 \mathrm{D}$ electromagnetic codes such as SUPERFISH and HFSS.

The required voltage per strip was calculated to be about $45 \mathrm{kV}$ and the uniformity of the deflecting field as a function of the horizontal coordinate is of the order of $\pm 2 \%$ over all the kicker horizontal aperture $( \pm 2.7 \mathrm{~cm})$ while it is less than $10 \%$ over $\pm 1 \mathrm{~cm}$ along the vertical coordinate.

No longitudinal and horizontal HOMs are trapped in the structure. Concerning the vertical beam coupling impedance, four HOM are trapped in the structure with a vertical impedance of the order of a few tens of $\mathrm{kV}$ per meter.

The kickers have been built and installed in the DAФNE collider and no problems occurred to them since they also demonstrate to be very versatile devices to be used in different operation conditions. Injection with the $45 \mathrm{KV}$ pulsers has been successfully tested but showed poor reliability. Other possible injection schemes have been tested successfully: with the old long pulse generators and in a hybrid configuration. Lower voltage $(25 \mathrm{kV})$ fast pulse generators have been successfully tested for two months and they will be finally used for injection in the next DAФNE run.

Low power $\mathrm{rf}$ measurements have been also done and compared with HFSS simulations showing very good agreement and a total comprehension of the device from the electromagnetic point of view.

A simple circuital model for tapered striplines has been also proposed to estimate the kicker efficiency and the beam transfer and longitudinal impedances.

\section{ACKNOWLEDGMENTS}

We would like to thank G. Sensolini for the technical support in kicker design and construction, S. Pella for the technical support in high voltage tests, M. Zobov for helpful discussion on stripline kicker properties, and A. Drago for help in the measurements with the transverse feedback system. This work is partially supported by the Commission of the European Communities under the 6th Framework Program "Structuring the European Research Area", Contract No. RIDS-011899.

[1] C. Milardi et al., in Proceedings of the 23rd Particle Accelerator Conference, Vancouver, Canada, 2009 (IEEE, Piscataway, NJ, 2009).

[2] M. Bazzi et al. (SIDDHARTA Collaboration), Phys. Lett. B 681, 310 (2009).

[3] M. Preger, DAФNE Technical Note I-2, Frascati, 1990.

[4] S. De Simone and A. Ghigo, DAФNE Accumulator Kickers, EPAC 92, Berlin, 1992.

[5] D. Alesini, S. De Simone, A. Gallo, A. Ghigo, and F. Marcellini, in Proceedings of the International Workshop on Performance Improvement of Electron-Positron Collider Particle Factories ( $e^{+} e^{-}$Factories'99), KEK Proc. 99-24 (KEK, Tsukuba, Japan, 1999), p. 146.

[6] http://www.fidtechnology.com/.

[7] D. Alesini et al., in Proceedings of the 10th European Particle Accelerator Conference, Edinburgh, Scotland, 2006 (EPS-AG, Edinburgh, Scotland, 2006), p. 1502.

[8] D. Alesini et al., Fast Injection Kickers for DAФNE and ILC Damping Ring, DAФNE Technical Note No. I-17, Frascati, 2006; EUROTeV-Report-2006-025, 2006, http:// www.eurotev.org/reports__presentations/eurotev_reports/ 2006/e668/EUROTeV-Report-2006-025.pdf.

[9] D. A. Goldberg and G. R. Lambertson, Report No. LBL31664 ESG-160, 1992.

[10] T. Naito, H. Hayano, M. Kuriki, N. Terunuma, and J. Urakawa, Nucl. Instrum. Methods Phys. Res., Sect. A 571, 599 (2007.

[11] B. I. Grishanov, F. V. Podgorny, J. Ruemmler, and V.D. Shiltsev, Nucl. Instrum. Methods Phys. Res., Sect. A 396, 28 (1997).

[12] John F. Power, Barbara Blind, and Andrew J. Jason, IEEE Trans. Nucl. Sci. 32, 3021 (1985).

[13] Report No. LBNL-59449, Cockroft-06-04, 2006, edited by A. Woslki, J. Gao, and S. Guiducci, available at http://www.linearcollider.org/pdf/20060511 _DRConfigRecommend.pdf.

[14] ILC Report Vol. III, edited by N. Phinney, N. Toge, and N. Walker, pp. 60-62 2007, available at http://www .linearcollider.org/about/Publications/Reference-DesignReport.

[15] James H. Billen, and Lloyd M. Young, Part. Accel. 7, 213 (1976) [http://laacg1.lanl.gov/laacg/services/ download_sf.phtml].

[16] www.ansoft.com. 
[17] F. Marcellini et al., in Proceedings of the 23rd Particle Accelerator Conference, Vancouver, Canada, 2009 (Ref. [1]).

[18] F. Caspers, in Handbook of Accelerator Physics and Engineering, edited by A.W. Chao and M. Tigner (World Scientific, Singapore-New Jersey-London-Hong Kong, 1999).

[19] M. Zobov, P. Arcioni, R. Boni, A. Gallo, A. Ghigo, F. Marcellini, M. Migliorati, L. Palumbo, L. Pellegrini, M. Serio, and B. Spataro, LNF Report No. 95/041(P), 1995.

[20] F. Marcellini et al., in Proceedings of the International Workshop on Performance Improvement of $e^{-} / e^{+}$
Collider Particle Factories (KEK, Tsukuba, Japan, 1999), p. 139.

[21] A. Drago, in Proceedings of the 23rd Particle Accelerator Conference, Vancouver, Canada, 2009 (Ref. [1]).

[22] www.gdfidl.de.

[23] B. R. Poole, G. J. Caporaso, Y.J. (Judy) Chen, and S.D. Nelson, in Proceedings of the 18th Particle Accelerator Conference, New York, 1999 (IEEE, New York, 1999), p. 1824.

[24] D. J. Martin, in IEEE Particle Accelerator Conference (APS Beam Physics, San Francisco, California, 1991). 